\title{
Supplementary DBEM
}

We provide details of the DBEM that are relevant to understand the outputs used in the subsequent ocean acidification socioeconomic analysis.

\section{Ecophysiological models}

Ocean acidification impacts were modelled with other stressors (i.e. temperature, dissolved oxygen) and uses a derived equation of the von Bertalanffy growth function (Tai, Harley, \& Cheung, 2018) to determine changes in growth rate, $B$, over time, $t$ :

$\frac{d B}{d t}=H W^{d}-k W^{b}$

where $H$ and $k$ represent the coefficients for anabolism and catabolism, respectively. The growth rate is a function of the oxygen supply (i.e. anabolism) and oxygen demand required for maintenance metabolism (i.e. catabolism). Anabolism scales with body weight $(W)$ to the exponent $d<1$. In this model, $d=0.7$, although values typically range from 0.5 and 0.95 . Sensitivity analyses showed that changes in temperature and acidity with low values of $d(<0.7)$ slightly decreases sensitivity, while larger values of $d(>0.7)$ markedly increases sensitivity (Pauly \& Cheung, 2017; Tai et al., 2018). The use of 0.7 is thus a conservative value as smaller values of $d$ only marginally decrease sensitivity to multiple stressors and larger values of $d$ only increases sensitivity. Catabolism scales linearly with $(W)$, i.e. $b=1$.

When maximum body size $\left(W_{\infty}\right)$ is reached and $d B / d t=0$, solving for equation (1) results in $H=$

$k W_{\infty}^{(1-d)}$. Then we can integrate equation (1) into a generalized von Bertalanffy growth function: 
$W_{t}=W_{\infty}\left[1-e^{-K\left(t-t_{0}\right)}\right]^{1 /(1-d)}$

where $K$ is the von Bertalanffy growth parameter where $K=k(1-d)$. The parameter $K$ represents the rate at which maximum body size is reached.

Environmental stressors are integrated to affect metabolism using:

$H=g\left[O_{2}\right] e^{-j_{1} / T}$

and

$k=h\left[H^{+}\right] e^{-j_{2} / T}$

$H$ and $k$ coefficients as a function of the Arhennius equation, $e^{-j / T}$, where $j_{1}$ and $j_{2}$ are equal to $E_{a} / R$ where $E_{a}$ (for anabolism and catabolism, respectively) and $R$ are the activation energy and Boltzmann constant, respectively. Constants $j_{1}$ and $j_{2}$ are equal to $E_{a} / R$ where $E_{a}$ (for anabolism and catabolism, respectively) and $R$ are the activation energy and Boltzmann constant, respectively, while T is the absolute temperature (in Kelvin) (Cheung, Dunne, Sarmiento, \& Pauly, 2011). Oxygen availability (i.e. dissolved oxygen concentration) and acidification (i.e. hydrogen ion concentration) affect aerobic scope changing oxygen supply (anabolism) and oxygen demand (catabolism), respectively. Our model assumes that multi-stressor impacts following the most viable and parsimonious current working hypothesis (oxygen- and capacitylimited thermal tolerance hypothesis), to link physiological responses to life history traits. Coefficients $g$ and $h$ from equations (3) and (4), respectively, were derived for each species from the average $W_{\infty}, K$, and environmental temperature $T_{0}$ reported in the literature (Cheung et al., 2011): 
$g=\frac{W_{\infty}^{(1-d)} K}{\left[O_{2}\right] e^{-j_{1} / T}}$

and

$h=\frac{K /(1-d)}{\left[H^{+}\right] e^{-j_{2} / T}}$

Changes in aerobic scope are linked to changes in the asymptotic weight $\left(W_{\infty}\right)$ and von Bertalanffy growth parameter $K$ :

$W_{\infty}=\left(\frac{H}{k}\right)^{1 /(1-d)}$

and

$K=k(1-d)$

Other parameters - e.g. asymptotic length and the length at maturity - that scale with weight can also be predicted, (Beverton \& Holt, 1959).

\section{Population dynamics models}

We modelled populations using an intrinsic population growth model (Hilborn \& Walters, 1992):

$G_{i}=r \cdot A_{i} \cdot\left(1-\frac{A_{i}}{K C_{i}}\right)$

where $G_{i}$ is the population growth in any given grid cell $i, r$ is the intrinsic rate of population increase, $A$ is the abundance, and $K C$ is the carrying capacity. Thus, changes in abundance was modelled as: 


$$
\frac{d A_{i}}{d t}=\sum_{j=1}^{N} G_{i}+L_{i j}+I_{i j}
$$

where $L$ and $I$ are the settled larvae and net migrated adults, respectively, from surrounding cells $j$ into focal cell $i$.

Carrying capacity was modelled to change as a function of habitat suitability, $P$, with the equations (Cheung, Lam, \& Pauly, 2008) :

$K C_{t+1}=K C_{t} \cdot\left(\frac{P_{t+1}}{P_{t}}\right)$

and

$P=P(T) \cdot P($ Dep $) \cdot P(H) \cdot P($ Ice $)$

where $T, D e p, H$, and Ice are corresponding temperature, depth, habitat type, and sea ice coverage for each cell, respectively. A major assumption of the model is that the each cell is at carrying capacity at the start of the simulation — the averaged 1951 to 1970 time period.

\section{References for S1 Note:}

Beverton, R. J. H., \& Holt, S. J. (1959). A review of the life span and mortality rate of fish in nature and the relation to growth and other physiological characteristics. In G. E. W. Wolstenholme \& M. O'Connor (Eds.), CIBA Foundation Colloquia on Ageing: the Lifespan of Animals (pp. 1422-177). Churchill, London: J. \& A.

Cheung, W. W. L., Dunne, J., Sarmiento, J. L., \& Pauly, D. (2011). Integrating ecophysiology and plankton dynamics into projected maximum fisheries catch potential under climate change in the Northeast Atlantic. ICES Journal of Marine Science, 68(6), 1008-1018. https://doi.org/10.1093/icesjms/fsr012 
Cheung, W. W. L., Lam, V., \& Pauly, D. (2008). Modelling present and climate-shifted distribution of marine fishes and invertebrates. (Fisheries Centre Research Reports No. 16(3)) (p. 72). University of British Columbia. Retrieved from http://hdl.handle.net/2429/40936

Hilborn, R., \& Walters, C. J. (1992). Quantitative Fisheries Stock Assessment. Springer, Boston, MA.

Pauly, D., \& Cheung, W. W. L. (2017). Sound physiological knowledge and principles in modeling shrinking of fishes under climate change. Global Change Biology, 24(1), e15e26. https://doi.org/10.1111/gcb.13831

Tai, T. C., Harley, C. D. G., \& Cheung, W. W. L. (2018). Comparing model parameterizations of the biophysical impacts of ocean acidification to identify limitations and uncertainties. Ecological Modelling, 385, 1-11. https://doi.org/10.1016/j.ecolmodel.2018.07.007 